\title{
Estratégia de ensino durante a pandemia de COVID-19 para profissionais da vigilância em saúde, Minas Gerais: um relato de experiência
}

\author{
Teaching strategy during the COVID-19 pandemic for health surveillance professionals, Minas
}

Gerais: an experience report

Estrategia de enseñanza durante la pandemia de COVID-19 para profesionales de la vigilancia de

la salud, Minas Gerais: un informe de experiencia

Recebido: 25/02/2021 | Revisado: 03/03/2021 | Aceito: 19/03/2021 | Publicado: 26/03/2021

\author{
Marcela Bella Lopes \\ ORCID: https://orcid.org/0000-0002-9761-5030 \\ Universidade Federal Fluminense, Brasil \\ E-mail: bellalps@hotmail.com \\ Benedito Carlos Cordeiro \\ ORCID: https://orcid.org/0000-0001-6387-511X \\ Universidade Federal Fluminense, Brasil \\ E-mail: bcordeiro@id.uff.br
}

\begin{abstract}
Resumo
A pandemia do novo Coronavírus trouxe desafios importantes não só do ponto de vista do cuidado ao paciente, mas também no campo do ensino-aprendizagem para que os profissionais da saúde conseguissem ter acesso a todas informações para um trabalho seguro e ágil diante de tantas orientações disponibilizadas em pouco tempo. Este estudo, de caráter descritivo, qualitativo, do tipo relato de experiência, foi desenvolvido entre março e abril de 2020, impulsionado pelas dúvidas que chegavam aos coordenadores de vigilância em saúde, epidemiologia e vigilância sanitária de uma regional de saúde de Minas Gerais (MG) quanto às temáticas de vigilância para atuação nos territórios. Assim, objetivou-se desenvolver uma estratégia educacional para quinze municípios de duas regiões de saúde em MG como proposta de educação permanente que fosse capaz de organizar documentos publicados em diversos sites, e superar a dificuldade na busca por informações na rotina do trabalho no início do enfrentamento da pandemia de COVID-19. Os resultados mostraram que a construção de uma estratégia de ensino, como o Guia de Orientações Técnicas nas Ações de COVID-19, motivada pelos trabalhadores da vigilância, favoreceu a organização das orientações técnicas prioritárias a serem usadas pelas equipes dos territórios, possibilitando a autonomia no trabalho e oportunizando ações.
\end{abstract}

Palavras-chave: Infecções por coronavírus; Vigilância em saúde pública; Educação continuada; Epidemiologia.

\begin{abstract}
The new Coronavirus pandemic brought important challenges not only from the point of view of patient care, but also in the field of teaching and learning so that health professionals could have access to all information for safe and agile work in the face of so many guidelines available soon. This descriptive, qualitative, experience report type study was carried out between March and April 2020, driven by the doubts that came to the coordinators of health surveillance, epidemiology and health surveillance of a regional Health in Minas Gerais (MG), regarding the themes of surveillance for action in the territories. Thus, the objective was to develop an educational strategy for 15 municipalities in two health regions in MG as a proposal for permanent education, which would be able to organize documents published on various sites and overcome the difficulty in searching for information in the work routine, at the beginning coping with the COVID-19 Pandemic. The results showed that the construction of a teaching strategy, such as the Guide to Technical Guidance in COVID-19 Actions, motivated by surveillance workers, favored the organization of priority technical guidelines to be used by teams in the territories, enabling autonomy at work, enabling actions.
\end{abstract}

Keywords: Coronavirus infections; Public health surveillance; Education continuing; Epidemiology.

\section{Resumen}

A pandemia do novo Coronavírus trouxe desafios importantes não só do ponto de vista do cuidado ao paciente, mas também no campo do ensino-aprendizagem para que os profissionais da saúde conseguissem ter acesso a todas informações para um trabalho seguro e ágil diante de tantas orientações disponibilizadas en poco tiempo. Este estudio descriptivo, cualitativo, tipo informe de experiencia se realizó entre marzo y abril de 2020, impulsado por las dudas que llegaron a los coordinadores de vigilancia en salud, epidemiología y vigilancia de la salud de una Salud regional en Minas Gerais (MG), en torno a los temas de vigilancia para la acción en los territorios. Así, el objetivo era 
desarrollar una estrategia educativa para 15 municipios de dos regiones de salud de MG como propuesta de educación permanente, que pudiera organizar documentos publicados en varios sitios web y superar la dificultad de búsqueda de información en la rutina laboral. al principio para hacer frente a la pandemia COVID-19. Los resultados mostraron que la construcción de una estrategia docente, como la Guía de Orientación Técnica en Acciones COVID-19, motivada por trabajadores de vigilancia, favoreció la organización de lineamientos técnicos prioritarios para ser utilizados por los equipos en los territorios, posibilitando la autonomía en el trabajo, habilitando acciones.

Palabras clave: Infecciones por coronavirus; Vigilancia en salud pública; Educación continua; Epidemiología.

\section{Introduçãa}

Desde a década de 80, a população brasileira tem convivido com algumas situações inusitadas nos sistemas de saúde com a reintrodução de doenças mundialmente controladas, podendo ser por alteração de virulência ou patogenicidade. Tais ocorrências geraram diversos efeitos epidêmicos de amplitude considerável, e causaram mudanças diretas no perfil epidemiológico, direcionando ações no campo da vigilância em saúde para exercer seu papel de proteção e prevenção oportuna, e controlando avanços de agravos à saúde da população (Teixeira, Costa, Carmo, Oliveira \& Penna, 2018).

As epidemias têm um significado próprio por acometer um grande número de pessoas e, singularmente, por ocorrer em um definido espaço de tempo, necessitando de intervenções baseadas no conhecimento científico e da atuação do Estado, marcando diversos momentos históricos, e ocasionando grande impacto no modo de viver e de superação da sociedade (Barata, 1987). As pandemias se diferenciam das epidemias porque ambas são causadas por doenças infecciosas e transmissíveis que começam em uma determinada região, contudo a primeira tem uma característica de se espalhar rapidamente em grandes proporções favorecida pela globalização (Ferreira et al., 2014).

O novo Coronavírus, nomeado por SARS-COV-2, que causa a COVID-19, foi detectado primeiramente em dezembro de 2019 na China. Em janeiro de 2020, a Organização Mundial de Saúde (OMS) confirmou a circulação do vírus nos Estados Unidos, Canadá e Austrália e, em fevereiro, no Brasil (Lana et al., 2020a). Devido à forma de transmissão ser essencialmente pessoa a pessoa, tanto por pacientes assintomáticos ou aqueles com sintomas respiratórios, até mesmo durante o período de incubação, muitas medidas de precaução e prevenção foram amplamente estudadas dada gravidade do vírus (Carvalho et al., 2020). As indicações de intervenções não farmacológicas, tais como, medidas ambientais de arejamento, higienização de superfícies e ambientes, medidas comunitárias de contenção da dispersão do vírus, representam grande desafio para que sejam utilizadas oportunamente de modo a evitar a subida abrupta da curva epidêmica, por isso a importância de cada medida corretamente realizada (Garcia \& Duarte, 2020). A primeira fase da pandemia é considerada como contenção, que antecede a segunda caracterizada por mitigação, quando a transmissão sustentada no país já se instalou, necessitando de medidas que consigam evitar que pessoas de risco contraiam os casos graves, sendo duas importantes etapas que necessitam de medidas que achatem a curva da epidemia (Werneck \& Carvalho, 2020).

No contexto da emergência em saúde pública de importância nacional, a vigilância em saúde tem um papel fundamental na tomada de medidas de prevenção oportuna, controlando e minimizando danos e agravos à coletividade, principalmente quando as epidemias apontam por abalar a capacidade de resposta do Sistema Único de Saúde (SUS), utilizando-se dos pontos focais da vigilância para o direcionamento das ações que serão fundamentais para suportar as demandas (Teixeira, Costa, Carmo, Oliveira \& Penna, 2018). O trabalho da vigilância em saúde é fornecer informação técnica constante e atual para os trabalhadores de saúde, ser capaz de realizar ações articuladas e integradas de acordo com as peculiaridades de cada cenário, proporcionando respostas efetivas para os problemas de saúde (Pinto, Pereira \& Limongi, 2017).

Vários esforços são colocados em prática a fim de acompanhar a dinâmica da vigilância em saúde internacional de maneira bastante sensível, aumentando o compartilhamento de informações, de descobertas e análises com o objetivo único de contenção (Lana et al., 2020b). Com a velocidade na disseminação do conhecimento via ferramentas tecnológicas, incluindo a 
rede de informação - em que a atualização do profissional se torna muito dinâmica -, torna-se fundamental propor novas tecnologias educacionais que favoreçam o ensino-aprendizagem individual, coletivo e institucional, demandando inovação na formação dos profissionais de saúde diante de situações de grande complexidade (Ceccim, 2005). As ferramentas disponibilizadas para a formação no SUS são, ainda, insipientes, mas com grandes chances de inovação tecnológica, materializadas pelo computador e permitindo o surgimento de novos processos criativos. Junto deles, é possível uma dupla construção natureza-sociedade, seja por meio dos objetos de aprendizagem (OA), seja pela Teoria do Ator Rede de Callon, em que seres humanos, artefatos tecnológicos, organizações, instituições, dentre outros, todos são os atores do processo (Cavalcante \& Vasconcellos, 2007).

Em Minas Gerais, a propagação não foi diferente, e nem a necessidade de rápidas respostas do sistema de saúde, necessitando de estratégias que fossem capazes de auxiliar o trabalho dos profissionais no campo da vigilância para execução de ações de saúde nos territórios. A publicação de inúmeros documentos para a atuação em vigilância em saúde foi surgindo à medida que o coronavírus foi avançando nos Estados, sendo importante organizá-los e selecioná-los para favorecer a atuação oportuna e adequada nos campos da prevenção. Seria possível uma técnica de educação permanente na área de ensino para que as equipes de saúde pudessem atuar no cenário da vigilância sanitária e epidemiológica com agilidade e segurança no trabalho?

Nesse contexto, este relato de experiência tem como objetivo apresentar o Guia de Orientações Técnicas nas Ações de Covid-19 como estratégia educacional disponibilizada aos municípios de duas regiões de saúde em Minas Gerais para atender as necessidades do trabalho da vigilância em saúde, vigilância sanitária e vigilância epidemiológica como proposta de educação permanente na fase de contenção e mitigação. Tal prática teve como objetivo organizar os documentos publicados em diversos sites e superar a dificuldade na busca por informações na rotina do trabalho relatadas pelos profissionais de saúde.

\section{Metodologia}

Trata-se de um estudo descritivo, qualitativo de natureza relato de experiência, que ocorreu no período de 20 de março a 15 de abril de 2020, direcionado a profissionais de saúde dos municípios de duas regiões de saúde em Minas Gerais. Estudos qualitativos precisam levar em consideração as opiniões que estão ligadas ao tema pesquisado, diferentemente de estudos quantitativos que serão representados por números em avaliações estatísticas (Spalding et al., 2020).

A partir da percepção dos coordenadores regionais de vigilância em saúde, vigilância sanitária e vigilância epidemiológica de que os municípios sob jurisdição estavam tendo muitas dúvidas em relação aos materiais técnicos a serem utilizados para responder às ações de vigilância no combate à COVID-19 nos territórios, foi identificada a necessidade de uma estratégia que facilitasse e pudesse agilizar o trabalho in loco.

As etapas do trabalho foram as seguintes:

$1^{\text {a }}$. Levantamento dos questionamentos recebidos por e-mail institucional, por telefone e no grupo de WhatsApp dos técnicos municipais sobres quais dúvidas eram mais frequentes e mais relevantes para o momento.

$2^{\circ}$. Seleção dos temas mais destacados no âmbito da vigilância sanitária: "alimentos, barreiras sanitárias, água e esgoto, desinfecção de cidades, hospital de campanha, hotéis, instituto de acolhimento, instituto de longa permanência para idosos, máscaras caseiras, máscaras para profissionais de saúde, medicamentos, óbitos, odontologia, produção de Equipamentos de Proteção Individuais (EPI), saneantes, serviços essenciais, serviços de saúde, sistema prisional, telemedicina e transporte sanitário". Na vigilância epidemiológica, os temas destacados foram: "arboviroses e COVID-19, compulsoriedade no enfrentamento da COVID-19, declaração de óbitos, influenza e COVID-19, protocolos, saúde do trabalhador, testes rápidos e testes rápidos em farmácia e drogarias".

$3^{\text {a }}$. Estudo nos sites do Ministério da Justiça e da Segurança Pública, do Ministério da Saúde (MS), da Agência Nacional de Vigilância Sanitária (Anvisa) e da Secretaria Estadual de Saúde de Minas Gerais (SES-MG) observando a 
vigência de cada informação.

$4^{\text {a }}$. Coleta dos documentos, mantendo as últimas versões e suas atualizações.

5a. Definição do formato do Guia de Orientações Técnicas nas Ações de COVID-19, atendendo aos critérios de facilidade visual, organização dos temas, ativação dos hiperlinks selecionados, definição de páginas para a construção.

\section{Resultados e Discussão}

A estratégia de educação permanente permitiu a construção do Guia de Orientações Técnicas nas Ações de COVID19, impulsionada pelos questionamentos dos trabalhadores de vigilância em saúde, epidemiológica e sanitária, favorecendo a organização dos documentos técnicos prioritários a serem usados pelas equipes dos quinze municípios de uma região de saúde em Minas Gerais. O Guia de Orientações Técnicas nas Ações de COVID-19 teve seu foco delimitado pelas ações apenas de vigilância em saúde em todo o seu contexto de atuação, não se estendendo para o campo da assistência ou regulação à saúde, pois as equipes que apresentaram as dificuldades para as coordenações estaduais eram trabalhadores dessa área.

O Guia possibilitou, ainda, a autonomia dos trabalhadores com o material em mãos para aplicação nas atividades diárias oportunizando ações. A aprendizagem deve ter um caráter significativo em que o dia a dia dos profissionais e as realidades vivenciadas devem ser considerados, possibilitando fazer uma ligação entre as situações-problema a fim de favorecer estratégias que possam contribuir com a prática, sendo capazes de proporcionar o aprendizado coletivo (Croda \& Garcia, 2020).

Urge compreender que é possível ter um olhar multidimensional além do que se costuma visualizar normalmente no campo do agir no espaço laboral. Desse modo, compreende-se, também, que o local de trabalho, por meio das práticas diárias, possibilita produção de conhecimento, destacando o encontro com o outro e a troca de modos de agir e saber, não sendo necessária a distinção entre trabalhador ou profissional de saúde para que se alcance sucesso, fruto dessa relação (Merhy, 2015a).

Foi possível produzir uma técnica de ensino capaz de disponibilizar documentos técnicos de instituições oficiais seguros, dando ressignificação às legislações para o trabalho no campo, e favorecendo a organização dos marcos regulatórios a serem utilizados na rotina. A busca de todos os temas apontados pelas equipes municipais foi feita em dois dias de modo a manter a atualização mais recente. No momento de pandemia, com o excesso de informações, o cuidado quanto a origem das informações era essencial, já que o quantitativo de divulgações pela internet aumentava a preocupação quanto a veracidade dos conteúdos no trabalho na saúde (Motta, Freitas, Júnior, Blatt \& Caregnato, 2021).

Com todo o material coletado, optou-se pela construção de fácil consulta organizado por temas, proporcionando direcionamento para a busca, evitando as dificuldades nas pesquisas na internet. O layout único para as nove páginas proporcionou a inclusão de novos assuntos em outras versões de modo que atualizações pudessem ocorrer periodicamente.

Promover a formação dos trabalhadores do SUS, identificando e transformando as necessidades em estratégias que qualificam as ações nos territórios, faz parte dos objetivos da educação permanente, contribuindo com o processo formativo dos profissionais (Brasil, 2018). Uma premissa importante que a educação permanente traz em sua essência é descortinar as práticas do trabalho diária, focando na resolução de problemas permitindo evolução, pensamento e inovação (Marcondes, Fonseca, Silveira \& Tavares, 2016). A utilização de recursos que possibilitam o ensino aos profissionais de saúde com objetos de aprendizagem contribui amplamente com a educação em saúde (Motta et al., 2021)

Com a construção do Guia de Orientações Técnicas nas Ações de COVID-19 finalizada, este foi disponibilizado para as equipes de vigilância das duas regiões de saúde do Estado, primeiramente pelo e-mail institucional. Foi, também, repassado por grupos de WhatsApp, nos quais a divulgação foi facilitada, sendo orientado que contribuições dos profissionais municipais poderiam ser consideradas para novas versões. Os conhecimentos produzidos e evidenciados precisam estar inseridos em 
grupos que os transformem. Além disso, deve haver sua utilização adequada, em que o saber científico pode ser aplicado nas tomadas de decisão de acordo com a necessidade de cada serviço de saúde (Bezerra, Felisberto, Costa \& Hartz, 2019).

É importante colocar, em um patamar diferenciado, os saberes atuais e, por meio de ferramentas, destacá-los para serem utilizados na prática cotidiana. $\mathrm{O}$ encontro com o outro e a identificação de suas necessidades são prerrogativas para favorecer a produção de conhecimento, sendo capaz de gerar uma ciência ambulante (Merhy, 2015b). A gestão do trabalho em saúde, principalmente das pessoas, deve considerar a estruturação do trabalho, a organização e a inserção desse profissional nas atividades que desempenha. Desse modo, envolve o seu problema cotidiano e o ressignifica com objetivo de aperfeiçoar o processo educativo (Silva, Soder, Petry \& Oliveira, 2017).

O Guia trouxe, como aprendizado, a possibilidade de organizar saberes utilizando-se de instrumentos nada complexos e de grande contribuição. É possível que o trabalho seja, a partir de novos processos, uma escola de formação sempre contínua no exercício de suas funções, permitindo a atualização de conhecimentos. A necessidade de formação vem do exercício profissional e deve ser aproveitada como ocorre pela educação permanente em serviços (Merhy, 2015a).

A experiência trouxe a possibilidade de poder contribuir no processo ensino-aprendizagem junto às equipes municipais que estão no enfrentamento direto da COVID-19. Permitiu, assim, auxiliar nas necessidades da rotina dos trabalhadores, quer sejam com as atividades sanitárias de apuração de denúncias, fiscalizações sanitárias, quer sejam com as notificações de pacientes suspeitos, orientação sobre manejos, testagem de pacientes, codificação de óbitos e outras situações dentro do escopo da vigilância. O impacto positivo, na utilização do Guia, impulsionou para a elaboração da sua segunda versão após quinze dias, sendo revisado em todos os seus assuntos, retirados aqueles considerados desnecessários e incluindo novos temas demandados pelas equipes municipais. A forma de organização do Guia favoreceu que outras instituições organizassem seus materiais para dispor aos trabalhadores uma proposta de organização didática, sendo possível que adaptações gerem novas pesquisas quanto à capacidade de disponibilizar documentos de acordo com a necessidade dos profissionais de saúde.

Uma grande concentração de esforços está centrada entre os pesquisadores para diferentes estudos ligados à dinâmica do vírus, sendo que a ciência e o conhecimento por meio da divulgação de novos relatos favorecem um número considerável de publicações em torno da temática capaz de auxiliar a saúde pública na solução dos problemas causados pelo novo coronavírus (Barbosa, 2020).

Ensino e Saúde precisam ser considerados, acima de tudo, como uma prática social a partir de uma proposta educativa que auxilie na construção dos profissionais em sujeitos de si mesmos, em que a estratégia estimule que cada um diminua a sua dependência e submissão e favoreça um processo ensino-aprendizagem capaz de atender o que se pensa, como o profissional interage ou age em sociedade (Tourinho \& Raimondi, 2010).

A limitação de um Guia em uma pandemia se dá pelo contexto dinâmico das publicações nacionais, mas não impede de se definir uma periodicidade de revisão. Pelo fato da divulgação ter ocorrido pelo e-mail institucional e WhatsApp dos servidores municipais, não foi possível avaliar o número de acessos ao Guia.

\section{Considerações Finais}

A técnica de ensino elaborada a partir da necessidade do trabalho no território, como estratégia de educação permanente, fez com que o Guia de Orientações Técnicas nas Ações de COVID-19 reduzisse os questionamentos quanto à localização das informações em sites pelos profissionais de saúde de duas regiões de saúde de Minas Gerais, trazendo agilidade e segurança no trabalho nos territórios.

É possível um instrumento de ensino-aprendizagem ser significativo e auxiliar na realidade do trabalho contribuindo com a ciência na possibilidade de novas pesquisas que possam colaborar com a formação em tempos de pandemia. 


\section{Referências}

Barbosa, A. C. Q. (2020). Lições sobre a Pandemia da COVID-19 e a Informação Científica. APS em Revista, 2(1), 70-72. https://doi.org/10.14295/aps.v2i1.60

Bezerra, L. C. A., Felisberto, E., Costa, J. M. B. da S., \& Hartz, Z. (2019). Translação do Conhecimento na qualificação da gestão da Vigilância em Saúde: Contribuição dos estudos avaliativos de pós-graduação. Physis: Revista de Saúde Coletiva, 29(1), e290112. https://doi.org/10.1590/s0103-73312019290112

Brasil. Ministério da Saúde. (2018). Política Nacional de Educação Permanente em Saúde: o que se tem produzido para o seu fortalecimento? Brasília: Ministério da Saúde.

Carvalho, A. R. V. S., Cezarotti Filho, M. L., Azevedo, P. C. P., Silveira Filho, R. N., Barbosa, F. T., Rocha, T. J. M., Sousa-Rodrigues, C. F., \& Ramos, F. W. S. (2020). Epidemiology, diagnosis, treatment, and future perspectives concerning SARS-COV-2: a review article. Revista da Associação Médica Brasileira, 66(3), 370-374. http://dx.doi.org/10.1590/1806-9282.66.3.370

Cavalcante, M. T. L., \& Vasconcellos, M. M. (2007). Tecnologia de informação para a educação na saúde: Duas revisões e uma proposta. Ciência \& Saúde Coletiva, 12(3), 611-622. https://doi.org/10.1590/S1413-81232007000300011

Ceccim, R. B. (2005). Educação Permanente em Saúde: Desafio ambicioso e necessário. Interface - Comunicação, Saúde, Educação, 9(16), 161-168. https://doi.org/10.1590/S1414-32832005000100013

Croda, J. H. R., \& Garcia, L. P. (2020). Immediate Health Surveillance Response to COVID-19 Epidemic. Epidemiologia e Serviços de Saúde, 29(1). https://doi.org/10.5123/s1679-49742020000100021

Ferreira, C. F., Dias, G. N., Franciscon, I. N., \& Oliveira, T. Q. (2014). Pandemias em um mundo globalizado: Desafios para o acesso universal à saúde. Brasília: UNB - Simulação das Nações Unidas para Secundaristas.

Garcia, L. P., \& Duarte, E. (2020). Intervenções não farmacológicas para o enfrentamento à epidemia da COVID-19 no Brasil. Epidemiologia e Serviços de Saúde, 29(2), e2020222. https://doi.org/10.5123/s1679-49742020000200009

Lana, R. M., Coelho, F. C., Gomes, M. F. da C., Cruz, O. G., Bastos, L. S., Villela, D. A. M., \& Codeço, C. T. (2020a). Emergência do novo coronavírus (SARS-CoV-2) e o papel de uma vigilância nacional em saúde oportuna e efetiva. Cadernos de Saúde Pública, 36, e00019620. https://doi.org/10.1590/0102$311 \times 00019620$

Lana, R. M., Coelho, F. C., Gomes, M. F. da C., Cruz, O. G., Bastos, L. S., Villela, D. A. M., \& Codeço, C. T. (2020b). Emergência do novo coronavírus (SARS-CoV-2) e o papel de uma vigilância nacional em saúde oportuna e efetiva. Cadernos de Saúde Pública, 36, e00019620. https://doi.org/10.1590/0102$311 \times 00019620$

Marcondes, F. L., Fonseca, P. I. M. N., Silveira, P. G., \& Tavares, C. M. M. (2016). Desvelando os sentidos da educação permanente em um programa municipal: estudo sociopoético. Journal Health NPEPS, 1(1), 40-52.

Merhy, E. E. (2015a). Educação Permanente em Movimento. Saúde em Redes, 1(1), 07-14. https://doi.org/10.18310/2446-4813.2015v1n1p07-14

Merhy, E. E. (2015b). Educação Permanente em Movimento-Uma política de reconhecimento e cooperação, ativando os encontros do cotidiano no mundo do trabalho em saúde, questões para os gestores, trabalhadores e quem mais quiser se ver nisso. Saúde em Redes, 1(1), 07-14. https://doi.org/10.18310/24464813.2015v1n1p07-14

Motta, L. D., Freitas, A. A. de, Júnior, R. X. J., Blatt, C. R., \& Caregnato, R. C. A. (2021). COVID-19 evidências para todos: Desenvolvimento de um objeto de aprendizagem no ensino em saúde [Preprint]. Revista Gaúcha de Enfermagem, 42, e20200281. https://doi.org/10.1590/1983-1447.2021.20200281

Pinto, D. S., Pereira, B. B., \& Limongi, J. E. (2017). Avaliação do conhecimento sobre Vigilância em Saúde en tre os profissionais do Sistema Único de Saúde, Uberlândia, Minas Gerais. Revista de Saúde e Ciências Biológicas, 5(1), 37-43. http://dx.doi.org/10.12662/2317-3076jhbs.v5i1.1046.p37-43.2017

Silva, L. A. A. da, Soder, R. M., Petry, L., \& Oliveira, I. C. (2017). Educação permanente em saúde na atenção básica: Percepção dos gestores municipais de saúde. Revista Gaúcha de Enfermagem, 38(1). https://doi.org/10.1590/1983-1447.2017.01.58779

Spalding, M., Rauen, C., Vasconcellos, L., Vegian, M., Miranda, K., Bressane, A. \& Salgado, M. (2020). Desafios e possibilidades para o ensino superior: uma experiência brasileira em tempos de COVID-19. Research, Society and Development, 9(8), e534985970. http://dx.doi.org/10.33448/rsd-v9i8.5970

Teixeira, M. G., Costa, M. da C. N., Carmo, E. H., Oliveira, W. K. de, \& Penna, G. O. (2018). Vigilância em Saúde no SUS - construção, efeitos e perspectivas. Ciência \& Saúde Coletiva, 23, 1811-1818. https://doi.org/10.1590/1413-81232018236.09032018

Tourinho, F. S. V., \& Raimondi, G. A. (2020). Ensino na Saúde em Tempos de Covid-19: Acesso, Iniquidades e Vulnerabilidade. Revista Brasileira de Educação Médica, 44(1), e164. http://dx.doi.org/10.1590/1981-5271v44.supl.1-editorial

Werneck, G. L., \& Carvalho, M. S. (2020). A pandemia de COVID-19 no Brasil: Crônica de uma crise sanitária anunciada. Cadernos de Saúde Pública, 36(5), e00068820. https://doi.org/10.1590/0102-311x00068820 